\title{
Arquitectura Orientada a Servicios, un enfoque basado en proyectos
}

\author{
Marcela Castro-León ${ }^{1}$, Francesc Boixader ${ }^{1}$, Dolores Rexachs², Emilio Luque ${ }^{2}$ \\ ${ }^{1}$ Escola Universitària d'Informàtica Tomàs Cerdà. \\ Sant Cugat del Vallès, Barcelona, España \\ \{marcela.castro, francesc.boixader\}@eug.es \\ ${ }^{2}$ Universidad Autónoma de Barcelona. \\ Barcelona, España \\ \{dolores.rexachs, emilio.luque\}@uab.es
}

Resumen. En este artículo se presenta el enfoque metodológico que se utiliza en la asignatura de Arquitectura Orientada a Servicios (AOS) que se imparte como obligatoria en el tercer curso del Grado de Informática y Servicios en la Escola Universitària d'Informàtica Tomàs Cerdà. AOS, es innovadora en un nivel educativo de grado, y propone, en la parte teórica de la metodología, enseñar las propiedades y características de la AOS de un modo conceptual relacionándolo con la tecnología existente. De este modo, los alumnos disponen de los criterios necesarios para valorar la oferta de productos que existen en el mercado actual, y adquieren conocimientos que van más allá de una línea de productos en concreto. En la parte práctica, se ha optado por un enfoque de aprendizaje basado en proyectos soportado por video-tutoriales, se consigue que el alumno proponga y desarrolle un proyecto basado en servicios web en tan solo una semana de uso de laboratorio. El método permite que el alumno aprenda, en forma consolidada y aplicada, los conceptos de tecnologías de servicios web SOAP, ReST y bus de servicios.

Palabras Clave: Arquitectura Orientada a Servicios, Servicios Web, Bus de servicios, SOAP, ReST

\begin{abstract}
In this paper we present the methodological approach we are using in Service Oriented Architecture (SOA), which is taught as a mandatory subject during the third course in Informatics and Services degree at the Escola Universitària d'Informàtica Tomàs Cerdà. This subject, innovative at grade level, proposes, in the theoretical part of its methodology, to teach the SOA properties and characteristics in a conceptual way and in relation to the current technology. As a result, the students are provided with the required elements to assess the current SOA product supply and they acquire the SOA knowledge beyond of a concrete product line. In the practical part, we choose project-based collaborative learning model supported with video-tutorial, we achieve the pupils could propose and develop a web services based project during using only a laboratory week. This method allows the students to learn in a practical way the technology concepts of SOAP, ReST, and service bus.
\end{abstract}

Keywords: Service Oriented Architecture, web services, Enterprise service bus, ESB, SOAP, ReST 


\section{Introducción}

El Grado de Informática y Servicios [1] que se imparte en la Escola Universitària d'Informàtica Tomàs Cerdà contempla una formación pluridisciplinar que abarca sectores tan diversos como el de servicios, el de las TIC o el financiero, y surge al detectar en el tejido económico de este país una demanda de profesionales en instituciones financieras y de seguros, gabinetes de asesoría científico-técnica, empresas con equipos interdisciplinarios de I+D y de transferencia de tecnología y, en el sector sanitario. Esta visión de acercar la informática al mundo de los servicios contiene un grado de originalidad, siendo esta propuesta pionera a nivel español.

Las AOS se han introducido en el mercado del software, por los beneficios relacionados que ofrecen a la hora de desarrollar aplicaciones distribuidas. El servicio es un componente de software asociado a un contrato o interfaz bien definida, y que se ejecuta en forma independiente del lenguaje en que fue creado y de la plataforma que lo provee o lo consume, utilizando protocolos estándares como HTML, XML o SOAP. Propone un acoplamiento orientado a mensajes con un mayor grado de autonomía que el de la computación tradicional de multiniveles, que facilita la reutilización, el diseño modular y por capas. El servicio se puede componer en servicios de mayor granularidad, con mayor nivel de abstracción hasta alcanzar una nivel adecuado para definir la gestión de procesos de una empresa y para aplicar seguridad de acceso y de confidencialidad de los datos. Además, esta arquitectura se adapta mejor a la nueva infraestructura, plataforma y servicios que ofrece Cloud Computing. El enfoque de AOS para los sistemas implica pensar y enseñar diferente considerando el objetivo de construir sistemas más efectivos [2].

Lawler y otros en [3] indican la importancia de introducir metodologías de enseñanza de AOS para apoyar el proceso de grandes inversiones en AOS que han realizado en los últimos años las empresas y que, sin embargo, falta un largo recorrido hasta lograr que las empresas sean Service Oriented Enterprise (SOE), es decir, que sus procesos de negocio estén completamente orientados a servicios.

La metodología de la asignatura AOS se basa, en la parte teórica, en transmitir al alumno las propiedades y características de AOS de modo conceptual y relacionarlo con la tecnología existente. De este modo, los alumnos disponen de los elementos necesarios para comprender las diferentes propuestas que existen en el mercado actual y, a su vez, adquieren conocimientos que van más allá de una línea de productos en concreto. En la parte práctica, se ha optado por un enfoque basado en proyectos utilizando herramientas de desarrollo con librerías y wizards altamente productivos y en video-tutoriales con los que el alumno puede desarrollar un proyecto basado en servicios web en una semana de práctica de laboratorio, trabajando en grupos de 2/3 personas. El método permite que el alumno aprenda en forma consolidada y aplicada los conceptos de tecnologías de servicios web SOAP, ReST y bus de servicios.

Este artículo presenta en la Sección 2, el contexto, el contenido y el enfoque general. En la Sección 3, se describen las competencias, los objetivos y el temario de la asignatura. En la Sección 4, se expone la metodología. En la Sección 5 se comentan otros enfoques de centros donde se estudia AOS. En la Sección 6 explicamos los resultados obtenidos y por último, en la Sección 7, enunciamos nuestras conclusiones. 


\section{La asignatura de Arquitectura Orientada a Servicios}

\subsection{Contexto}

La Escola Universitària d'Informàtica Tomàs Cerdà, adscrita a la Universidad Autónoma de Barcelona, elaboró un plan de estudios para el Grado de Informática y Servicios [1] que se incorporó desde el curso 2004-05 al plan piloto de la Universidad Autónoma de Barcelona para la adaptación al Espacio Europeo de Educación Superior y que se empezó a impartir en el curso 2009-10.

El perfil profesional objetivo que se puede adquirir mediante este grado se ajusta al de un ingeniero informático, dado que integra competencias en tecnología e ingeniería, que son complementadas con una formación más específica en el área de la gestión y organización empresarial, así como conocimientos, tanto en ciencias de la computación, comunicaciones y tecnologías web y multimedia. Este profesional debe ser capaz de evaluar, diseñar e implementar los sistemas de información en los que la tecnología juega un papel clave, con el objeto de gestionar la información utilizada por las empresas de servicios en todas sus áreas de negocio, en particular, aquellas que ofrecen los servicios a través de Internet, y, para hacerlo de manera óptima, tienen en cuenta la experiencia del usuario interno y externo a la empresa.

La asignatura AOS se imparte en el tercer año del grado en Informática y Servicios y está incluida en uno de los cinco bloques de contenidos del grado, en concreto en el de Servicios. AOS es una de las dos asignaturas obligatorias de la primera materia del bloque.

AOS es una asignatura que no aparece, como tal, en los planes de estudios de Grado de Informática, lo que hace que represente un elemento diferencial del Grado de Informática y Servicios con respecto al resto de ingenierías informáticas. AOS representa un modelo de integración entre software y empresa, concepto que es considerado clave en el desarrollo de este grado.

\subsection{Contenidos principales de la asignatura}

Los contenidos básicos de la asignatura son los siguientes:

- Arquitectura orientada a servicios.

- Arquitecturas de negocio y arquitectura orientada a servicios (SOA).

- Fundamentos del modelo de referencia de la organización de estándares de sistemas de información (OASIS).

- Orquestación en la arquitectura orientada a servicios.

- Bus de servicios de empresa y procesador de eventos inteligente.

- Lenguajes de especificación de servicios multiusuario, seguros y reutilizables.

- Metodologías de desarrollo de aplicaciones de servicios.

- Despliegue, mantenimiento y test de sistemas de gestión de procesos de negocio (BPMS).

Podemos distinguir cuatro pilares en el temario. En primer lugar, el concepto de AOS como arquitectura de negocio y de software. Luego, la definición de estándares, 
fundamentales para la comunicación de procesos en forma independiente de la tecnología y los lenguajes que los implementa. En tercer lugar, el bus de servicios, una de las piezas claves de AOS, es el que facilita la integración de los estándares con múltiples puntos de acceso y permite orquestar, procesar, registrar y controlar los diferentes eventos en la operación diaria de una empresa. Por último, la gestión de procesos de negocio, conocida por Business Process Management (BPM), es la cara visible de una empresa con AOS. BPM saca provecho a la arquitectura ofreciendo herramientas que permiten adaptar y desplegar cambios de los procesos de negocio en forma eficiente.

\subsection{Un enfoque conceptual y práctico}

El perfil del profesional del graduado en Ingeniería y servicios está pensado para ofrecer las nuevas habilidades tecnológicas que requieren las empresas del sector TIC del siglo XXI. Presenta una orientación profesional, y se alinea de la forma más efectiva posible con las necesidades del mercado laboral.

Por tanto, es necesario que el estudiante se familiarice con las tecnologías emergentes. No obstante, es necesario seleccionar aquellas que provienen de grupos de empresas reconocidas y/o que, pudiendo venir de grupos de open-source, utilicen estándares con un alto grado de aceptación por parte de la comunidad usuaria para dotar a los alumnos de habilidades no efímeras como pueden serlo algunas de las tecnologías emergentes que implementan AOS.

Durante la asignatura se enseña a diferenciar entre los conceptos teóricos relativos a la AOS y las propiedades de los productos de software disponibles en el mercado en la actualidad. Así entonces, el alumno desarrolla criterios que le permite reconocer con mayor facilidad las capacidades de un producto en concreto respecto al modelo teórico. Se pretende dotar a los alumnos de un conocimiento conceptual que tiene una mayor duración que el uso de una tecnología específica.

\section{Diseño de la asignatura}

\subsection{Competencias}

Del conjunto de competencias asociadas al grado de Informática y Servicios, la asignatura AOS selecciona las siguientes competencias específicas:

1. Evaluar sistemas hardware/software en función de un criterio de calidad determinado.

2. Adaptar la tecnología que sea realmente eficiente en su papel de intermediario en el proceso comunicativo entre las empresas de servicios y los usuarios de dichos sistemas.

3. Analizar, modelar y optimizar el conjunto de los procesos involucrados en los servicios, antes y después de su implementación. 
4. Gestionar la colaboración de los diferentes "actores" involucrados en la definición, diseño, implementación y explotación de servicios, así como integrarlos con las distintas tecnologías y metodologías.

De entre las competencias transversales asociadas al grado, la de Gestionar (planificar) el tiempo y los recursos disponibles, es la que en mayor grado se trabaja, por parte del alumnado, en el contexto de AOS.

\subsection{Objetivos}

A continuación se describen los objetivos que cumplen los alumnos que cursan con éxito la asignatura AOS, a través de los cuales se desarrollan las competencias mencionadas.

- Definir la arquitectura orientada a servicios, sus componentes en cada una de las capas de software (plataforma SOA) y las relaciones entre ellos.

- Conocer las propiedades de las aplicaciones orientadas a servicios, sus beneficios y los desafíos de implantar SOA en una empresa.

- Definir las objetivos particulares y características de cada uno de los componentes de la arquitectura orientada a servicios.

- Analizar productos de mercado AOS open-source y comerciales.

- Conocer las tecnologías y estándares más utilizados en la arquitectura SOA.

- Diseñar y desarrollar aplicaciones basadas en servicios web basados en protocolo SOAP y en la arquitectura ReST.

- Utilizar un Bus de servicios web para implementar ruteo y enmascaramiento de servicios web.

\subsection{Contenidos}

El programa de contenidos de la asignatura contempla los siguientes temas:

Tema 1.- Introducción a la Arquitectura Orientada a Servicios.

1.1. Definición, objetivos, fundamentos.

1.2. Plataforma tecnológica de SOA

1.3. Propiedades de SOA

Tema 2.- Tecnología de servicios web.

2.1. Estándares de servicios web.

2.2. Modelo de servicios web basados en SOAP.

2.3. Modelo de servicios web Representational State Transfer (ReST).

2.4. Diferencias, ventajas y desventajas entre modelos de WS SOAP y ReST.

Tema 3.- Productos SOA

3.1 Productos SOA Open Source para cada componente SOA

3.2 Establecimiento de criterios de selección según los objetivos empresariales.

3.3 Estudio de productos SOA comerciales.

Tema 4.- Composición de servicios WEB 
4.1 Definición, fundamentos de Service Component Architecture.

4.2 Principales elementos de Service Component Definition Language (SCDL).

Tema 5.- Integración de aplicaciones Enterprise Service Bus (ESB)

5.1 Definición y fundamentos del bus de servicios

5.2 Principales funcionalidades.

5.3 Implementaciones ESB más utilizadas en el mercado actual.

Tema 6.- Procesamiento de flujos de eventos Event Stream Processing (ESP)

6.1 Definición y fundamentos de ESP.

6.2 Estudio de la implementación Esper

Tema 7.- Gestión de procesos de negocio Business Process Manager (BPM)

7.1 Orquestación de servicios con BPEL.

7.2 Principales lenguajes estándares BPEL, BPMN i JPDL.

Este programa está diseñado para ser desarrollado en un semestre académico que comprende 16 semanas de clases teóricas, a razón de 3 horas de clase semanales, y otras 3 semanas de trabajo práctico en el laboratorio, con un total de 18 horas, bajo la directa supervisión del profesorado de la asignatura.

\section{Metodología docente}

\subsection{Clases teóricas}

La metodología utilizada para la enseñanza de los conceptos es top-down, en el sentido de que en el primer tema se da una visión completa de la arquitectura AOS, y en el resto, se desarrolla cada uno de los componentes profundizando en los detalles de los mismos. Esta metodología facilita la comprensión del temario por parte del alumno al incorporarlo de forma relacionada con el concepto integrador de AOS.

En la literatura se encuentran diferentes visiones o enfoques de AOS. La que hemos adoptado en la asignatura es considerar que representa una arquitectura de software abierta, ágil, extensible, federada y combinada, constituida por servicios autónomos, capaces de gestionar la calidad del servicio, posiblemente de diferentes proveedores, inter-operativos, potencialmente reutilizables e implementados todos ellos como servicios web [4]. Los principales beneficios que promete la adopción de esta arquitectura es simplificar el desarrollo y la implementación, reutilizar software, aprovechar mejor el gasto en servicios de computación, dar un criterio para la toma de decisiones y guiar las acciones de la empresa y, en particular, del departamento de IT.

En primera instancia, se desarrolla el concepto de AOS a través de la plataforma de componentes en sus diferentes niveles. En la figura 1, en la parte izquierda, se representan dichos niveles, comenzando por los servicios web que se ubican por encima de los sistemas legacy de la empresa. El siguiente nivel está formado por los servicios de negocio que se generan por composición de web-services (WS), siguiendo luego con los procesos de negocio (BPM) y la capa de presentación. En la parte derecha de la figura 1, se muestran los componentes que AOS añade a la infraestructura, como son el bus de servicios, el registro, las reglas de negocio, la monitorización y la seguridad. La bibliografía básica de referencia utilizada para el 
desarrollo de la plataforma AOS son los libros de Jeff Davis [5] y el de Juric [6]. Se comprende de este modo a la arquitectura AOS en forma global con sus propiedades y ventajas, por estar basada en servicios web y en comunicaciones estándares.

Luego, durante el transcurso del semestre, el alumno estudia en mayor profundidad cada uno de los componentes de la plataforma tecnológica sin perder la visión holística que se ha dado en un primer momento.

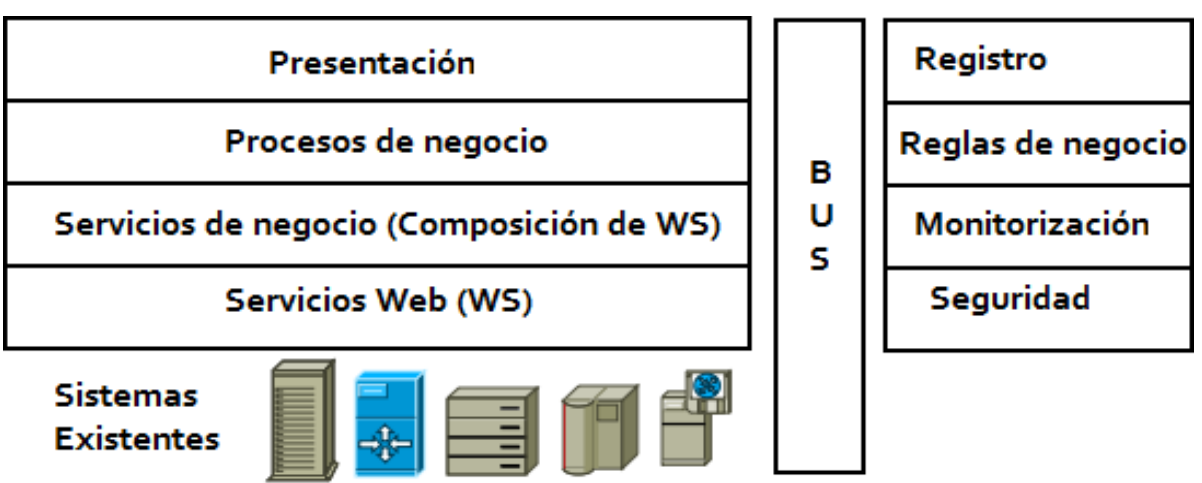

Figura 1. Plataforma tecnológica AOS [6].

\subsection{Clases prácticas - Metodología}

La metodología de las clases prácticas sigue las líneas del aprendizaje colaborativo basado en la elaboración de proyectos [7].

Durante el transcurso del semestre, se destinan tres semanas para las clases prácticas en el laboratorio que denominaremos 1, 2 y 3 . Estas semanas se ubican entre las 8 y 17 de las 19 que normalmente tiene un semestre. Los alumnos trabajan en grupos de $2 / 3$ personas, y durante dicha semana desarrollan un proyecto relacionado con el tema objetivo de cada práctica. A la semana siguiente, entregan y presentan los trabajos realizados.

Para que los alumnos dispongan de la información necesaria para llevar a cabo los proyectos de estas prácticas, se utiliza el blog Koonek [8], creado para esta asignatura. El blog está basado en videos-tutoriales que enseña al alumnado ejemplos de desarrollo de servicios web utilizando una plataforma basada en Netbeans [9], [10], Glassfish [11] y Java [12]. Esta plataforma ha sido seleccionada porque es una de las más consolidadas y desarrolladas en el uso de servicios web, y adoptada por un gran número de usuarios. Ha sido una de las pioneras en presentar implementaciones de servicios web para el desarrollo de aplicaciones. Esta vanguardia ha hecho que Netbeans disponga en la actualidad de un conjunto de opciones que facilitan y automatizan la conversión de una clase en servicio web y la creación de consumidores de estos servicios.

Las prácticas 1 y 2 son de servicios web, de SOAP [13] y ReST [14], respectivamente. Cada grupo ha de presentar una propuesta de trabajo práctico a desarrollar, a más tardar, una semana antes de la práctica. Dicha propuesta ha de 
incluir, al menos, un proveedor y un consumidor de servicio web. La propuesta se discute con el profesor para asegurar que el nivel de complejidad sea el adecuado para completar el trabajo en el tiempo asignado. Los servicios web proveedores tienen que incluir gestión de bases de datos o, bien uso de estructuras de datos complejas. La siguiente tabla 1 muestra cada una de las prácticas, indicando el objetivo y las habilidades que los alumnos tienen que haber adquirido antes del inicio de cada una.

Tabla 1. Relación entre práctica, objetivo y habilidades requeridas

\begin{tabular}{ccc} 
Práctica & Objetivo & Habilidades requeridas \\
\hline 1 & $\begin{array}{c}\text { Servicios WEB basados } \\
\text { en SOAP }\end{array}$ & $\begin{array}{c}\text { Creación de servicios web } \\
\text { SOAP proveedores y } \\
\text { consumidores. }\end{array}$ \\
\hline 2 & Servicios WEB basados & Creación de servicios web \\
en ReST & $\begin{array}{c}\text { ReST proveedores y } \\
\text { consumidores. }\end{array}$ \\
\hline 3 & Bus de servicios & $\begin{array}{c}\text { Manejo de la herramienta } \\
\text { de bus seleccionada }\end{array}$ \\
\hline
\end{tabular}

\subsection{Clases prácticas - Desarrollo}

Para la práctica 1 de WS SOAP se utiliza la especificación de java JAX-WS [15] y para la práctica 2, hemos adoptado el uso de la especificación JAX-RS [16], aunque en los servicios WS ReST también puede utilizarse XML y librerías de HTTP.

La instalación y configuración por defecto de las herramientas seleccionadas disponen de las librerías necesarias para el desarrollo de los servicios web. Esta característica, sumada a la provisión de wizard en Netbeans, permite que los alumnos desarrollen y desplieguen aplicaciones en forma muy rápida y sin errores, experimentando así los conceptos aprendidos, con un alto grado de satisfacción.

Los ejemplos que se presentan en el blog aumentan, gradualmente, el grado de complejidad hasta llegar al nivel que se les requiere en la propuesta a realizar.

En las clases anteriores a la entrega de propuesta, se presentan, comentan y discuten los video-tutoriales. Los alumnos disponen de la plataforma de desarrollo en sus máquinas, al menos uno por grupo, lo que les permite practicar en forma autónoma y/o en grupo. De este modo, los alumnos aprenden a utilizar las herramientas y a desarrollar WS en base a los ejemplos del blog.

La práctica 3 se dedica al uso de bus de servicios. Esta herramienta es de uso altamente recomendable en proyectos AOS debido a que permite integrar productos y servicios de diferentes tecnologías, centralizando en un lugar las políticas de gestión de diferentes protocolos y formatos de intercambio de datos, así como la política de ruteo de servicios, colas de mensajes, distribución y balanceo de carga, niveles de seguridad, control de accesos y auditoría. Para el desarrollo de esta práctica, se utiliza 
el producto Oracle Service Bus [17] ampliamente conocido en el sector empresarial. Este aplicativo trabaja en conjunto con el servidor de aplicaciones WebLogic.

Hemos seleccionado este producto porque provee un conjunto de tutoriales que explican, paso a paso, la implementación de un escenario para una empresa de préstamos de capital que muestra cómo realizar ruteo, transformación y validación de servicios [18]. Además dispone de un procedimiento de instalación que, aunque de una complejidad media, se encuentra lo suficientemente consolidado y con el soporte necesario para llevar a cabo las instalaciones en el laboratorio sin mayores incidencias.

La tabla 2 muestra los video-tutoriales que se publican en el blog en el orden que son presentados a los alumnos antes de cada práctica. La práctica 3 utiliza tutoriales en papel disponibles de la página de Oracle [18].

Tabla 2. Ejemplos tutoriales publicados en el blog para las práctica 1 y 2

\begin{tabular}{clll} 
Práctica & Ejemplos & Propósito didáctico \\
\hline 1 & $\begin{array}{l}\text { Creación de } \\
\text { WS SOAP } \\
\text { proveedor }\end{array}$ & $\begin{array}{l}\text { Procedimiento de desarrollo de un WS SOAP, } \\
\text { despliegue y test a partir de una clase java. }\end{array}$ \\
\hline 1 & $\begin{array}{l}\text { Creación de } \\
\text { WS SOAP } \\
\text { consumidor }\end{array}$ & $\begin{array}{l}\text { Creación de un programa java en web y cliente } \\
\text { pesado que invoque al WS proveedor creado por el } \\
\text { usuario o de uso público. }\end{array}$ \\
\hline 1 & $\begin{array}{l}\text { Proyectos de } \\
\text { años } \\
\text { anteriores }\end{array}$ & $\begin{array}{l}\text { Proyectos con WS proveedores con estructuras de } \\
\text { datos complejas o manejo de base de datos y } \\
\text { consumidores de WS embebidos en programas con } \\
\text { interfaz de usuario basadas en java script, servlets y/o } \\
\text { Java Server Faces. }\end{array}$ \\
\hline 2 & $\begin{array}{l}\text { Creación de } \\
\text { WS ReST } \\
\text { proveedor. }\end{array}$ & $\begin{array}{l}\text { Procedimiento de desarrollo de un WS proveedor } \\
\text { ReST. }\end{array}$ \\
\hline 2 & $\begin{array}{l}\text { Creación de } \\
\text { WS SOAP } \\
\text { consumidor }\end{array}$ & $\begin{array}{l}\text { Creación de una interfaz HTML5 que permita } \\
\text { invocar a un WS ReST. }\end{array}$ \\
\hline 2 & $\begin{array}{l}\text { Proyectos de } \\
\text { años } \\
\text { anteriores }\end{array}$ & $\begin{array}{l}\text { Proyectos que integren WS ReST proveedores y } \\
\text { consumidores entregados los años anteriores. }\end{array}$ \\
\hline
\end{tabular}

Los documentos a entregar al final de cada práctica (proyecto) incluyen:

- Memoria de contenido: Tiene que incluir la propuesta, objetivo, conceptos teóricos relacionados, mapa de arquitectura de la solución, desarrollo del trabajo, forma de instalación y despliegue y conclusiones.

- Presentación: Cada grupo presenta el trabajo realizado en cada práctica.

- Autorización para la publicación del trabajo. Cada alumno entrega su consentimiento para la publicación del trabajo práctico en el blog. 


\subsection{Método de evaluación}

El modelo de evaluación de la asignatura AOS consiste de:

- Primer Examen: $15 \%$. Se realiza antes de la semana 8 para que los alumnos estudien los conceptos de la plataforma AOS que se desarrollan en la primera parte.

- Examen Final: 25\%. Examen teórico de toda la asignatura al final del semestre.

- Prácticas: $30 \%$. (10\% cada una).

- Asistencia y actitud: $10 \%$

- Informes de prácticas: $10 \%$

- Presentaciones orales: $10 \%$

Se ha optado por otorgar idéntico peso a la teoría y a la práctica, -ya que el $10 \%$ de diferencia corresponde a las presentaciones orales que se refieren a las prácticas grupales pero que se valoran en forma individual-, lo cual representa un incentivo para que los alumnos dediquen un esfuerzo adicional alas prácticas de la asignatura..

\section{AOS en otros centros educativos}

La asignatura AOS se imparte en la Universidad de Granada, pero a nivel de Posgrado [19]. En dicha propuesta, AOS es visto como una arquitectura de desarrollo de WS y no como una plataforma general de infraestructura y software empresarial.

En la Universidad de La Laguna, en el posgrado en informática para la obtención del Máster Universitario en informática y se incluye la asignatura Arquitectura Orientada a Servicios [20]. El temario propuesto es similar al de nuestra asignatura, pero el enfoque se basa en que el alumno diferencie bien tres roles, como son el de analista de negocios, arquitectos de software y desarrolladores/ programadores.

La Universidad North Carolina State University en el grado de Computer Sciences imparte el curso de Service-Oriented Computing [21]. En dicho curso se enfatiza en conceptos, teorías y técnicas de servicios web a alto nivel. No se relaciona con la orientación a los procesos de negocio.

La Universidad de California en Berkeley School of Information, ofrece un conjunto de cursos específicos [22] en los cuales incluye los cursos de design and development of web-based products and services y Web-based Services, en los cuales se cubre parte del temario de nuestra asignatura, pero con un enfoque basado al desarrollo de aplicaciones.

La Universidad de Manchester, en School of Sciences, ofrece un proyecto de postgrado Postgraduate Research Projects denominado Mapping Applications and Services on Computing Clouds and Service-Oriented Systems [23]. En este proyecto se usan los sistemas orientados a servicios en lugar de la computación paralela tradicional por considerarla más dinámica donde múltiples servicios pueden estar ejecutándose.

En la Universidad de Warwick, en UK Manufacturing Group (WMG), que es el departamento de la facultad de ciencias dedicado a ofrecer cursos de Máster en tecnología e industria, imparte un curso denominado Service Management and Design 
[24] . En dicho curso se imparte una formación orientada a los líderes de funciones de servicio de una variedad de industria, orientado a aprender los sistemas y las técnicas usadas para gestionar servicios y creación de valor en los negocios y en los procesos.

\section{Resultados Obtenidos}

La metodología del aprendizaje basado en proyectos para las prácticas se ha utilizado desde que se comenzó a impartir la asignatura para fomentar el aprendizaje autónomo y en grupo, la gestión del tiempo y los recursos disponibles el uso. Sin embargo, en el último curso académico 2013/2014, hemos incorporado el uso de blog con videotutoriales como herramienta de soporte principal para los grupos de alumnos que encontraban dificultades durante el desarrollo de proyectos basados en servicios web. Se presentaban muchos errores, generalmente causados por un mal uso de las herramientas. El video-tutorial ayuda a mostrar cómo se han de utilizar correctamente. Al final del semestre, se realizó una encuesta, para saber la valoración del blog por parte de los alumnos, en la que preguntó ¿Cómo valoras el hecho que se disponga de un blog con ejercicios resueltos y trabajos prácticos anteriores?. En una respuesta de 1 a 5, (5 es la máxima valoración), se obtuvo una valoración media de 4, es decir, un $80 \%$. Sin embargo, hemos observado que en la ejecución de las prácticas, los alumnos realizaron propuestas que pueden adaptarse a proyectos existentes, y luego copiaron el código de los proyectos en las partes que podían, muchas veces sin una adaptación correcta. Para evitar este comportamiento, proponemos, por un lado, en el momento de la validación de la propuesta, se alerta sobre el parecido a un proyecto existente. Y por otro lado, se comunicará a los grupos la penalización grave en la nota en caso de encontrar copias de código que no corresponda a una correcta reutilización y adaptación de servicios.

\section{Conclusiones}

Se ha presentado la metodología actual de la asignatura Arquitectura Orientada a Servicios que se dicta en el tercer curso del Grado de Informática y Servicios de la Escuela Universitaria de Informática Tomàs Cerdà. La propuesta se basa en que los alumnos, en el transcurso del semestre, tengan una visión completa del potencial de la AOS para el desarrollo de software y de cómo esta arquitectura impacta en la gestión que la empresa realiza de un software integrado entre todos los sectores y departamentos. Una vez superada la asignatura, los alumnos son conocedores de la reutilización de los servicios y de su integración en aplicaciones independientemente de su tecnología y localización. AOS se convierte en un modelo altamente recomendable para que las empresas migren sus aplicaciones al paradigma del Cloud computing, debido a que por medio del servicio se puede administrar mejor la seguridad y realizar un uso más eficiente de recursos. 


\section{Referencias}

1. Boixader, Francesc [et al.]. El Grado en Informática y Servicios. Una respuesta a la nueva demanda del contexto social. A: JENUI 2010. "XVI Jornadas de Enseñanza Universitaria de la Informática". Universidade de Santiago de Compostela. Escola Técnica Superior d'Enxeñaría, 2010, p. 130-135..

2. B. A. Maurizio, J. Sager, P. Jones, G. Corbitt, and L. Girolami, "Service Oriented Architecture: Challenges for Business and Academia 3 . The Role of Business Process Management in SOA," pp. 1-8, 2008.

3. J. P. Lawler, H. Howell-barber, D. Colton, and K. A. Grant, "Critical Success Factors in the Planning of a Service-Oriented Architecture ( SOA ) Strategy for Educators and Managers," vol. 7, no. 94, 2009.

4. T. Erl, Service-oriented architecture: concepts, technology, and design. Pearson Education India, 2005.

5. J. Davis, Open Source Soa. Manning Publications Co., 2009.

6. M. B. Juric, SOA Approach to Integration: XML, Web Services, ESB, and BPEL in Realworld SOA Projects (Google eBook). 2007.

7. B. Antoni and G. Consuelo, "Incorporación de las TIC en la enseñanza y el aprendizaje basados en la elaboración colaborativa de proyectos," RUSC. Univ. Knowl. Soc. J., vol. 3, pp. 42-54, 2006.

8. "Koonek." [Online]. Available: http://koonek.blogspot.com.es/.

9. "Netbeans." [Online]. Available: http://www.netbeans.org.

10. H. Böck, The Definitive Guide to NetBeansTM Platform 7. Berkeley, CA: Apress, 2011.

11."Glassfish." [Online]. Available: http://www.glassfish.org.

12."Java." [Online]. Available: http://www.java.com/.

13.N. Mitra, Y. Lafon, and others, "Soap version 1.2 part 0: Primer," W3C Recomm., vol. 24, p. 12, 2003.

14.R. T. Fielding and R. N. Taylor, "Principled design of the modern Web architecture," ACM Trans. Internet Technol., vol. 2, no. 2, pp. 115-150, May 2002.

15.A. P. I. Java, "for XML-based web services (JAX-WS) 2.0.”.

16.M. Hadley and P. Sandoz, "JAX-RS: JavaTM API for RESTful Web Services," 2008.

17."Oracle Service [Online]. Aus." Available: http://www.oracle.com/technetwork/middleware/service-bus/overview/index.html.

18."Oracle Service Bus Tutoriales." [Online]. Available: https://java.net/downloads/oraclesoasuite11g/OSB/osb_examples_tutorials_111130.pdf.

19.P. García-Sánchez, M. Á. López Gordo, P. Castillo Valdivieso, J. González Peñalver, and M. I. García Arenas, "Web 2.0: Arquitectura orientada a servicios en Java," 2011.

20.E. S. Nielsen, "Arquitecturas orientadas a servicios: EEES, metodología docente y primeros resultados,” Jornadas Enseñanza Univ. la Informática.

21."North Carolina State University-Computer Sciences- Service-Oriented Computing." [Online]. Available: http://www.csc.ncsu.edu/faculty/mpsingh/local/SOC/s13/.

22."University of California, Berkeley School of Information." [Online]. Available: http://www.ischool.berkeley.edu/courses/i290.

23.M. Stubbs and P. Range, "Service-oriented architecture and curriculum transformation at Manchester Metropolitan University," Campus-Wide Inf. Syst., vol. 28, no. 4, pp. 299-304, 2011.

24."Warwick University, UK Manufacturing Group (WMG)." [Online]. Available: http://www2.warwick.ac.uk/fac/sci/wmg/education/wmgmasters/courses/masters_services_ management_design/. 